\title{
Use of caffeinated substances and risk of crashes in long distance drivers of commercial vehicles: case-control study
}

\author{
(c) $(1)$ (8)
}

\author{
Lisa N Sharwood research scholar ${ }^{1}$, Jane Elkington project manager ${ }^{1}$, Lynn Meuleners director ${ }^{2}$, \\ Rebecca Ivers injury division director ${ }^{1}$, Soufiane Boufous senior research fellow ${ }^{13}$, Mark Stevenson \\ director $^{4}$
}

${ }^{1}$ George Institute for Global Health, PO Box M201, NSW 2050, Australia; ${ }^{2}$ Curtin-Monash Accident Research Centre, WA 6845, Australia; ${ }^{3}$ Transport and Road Safety (TARS) Research, University of New South Wales, NSW, Australia; ${ }^{4}$ Monash University Accident Research Centre, Monash Injury Research Institute, VIC 3800, Australia

\begin{abstract}
Objective To determine whether there is an association between use of substances that contain caffeine and the risk of crash in long distance commercial vehicle drivers.

Design Case-control study.

Setting New South Wales (NSW) and Western Australia (WA), Australia.

Participants 530 long distance drivers of commercial vehicles who were recently involved in a crash attended by police (cases) and 517 control drivers who had not had a crash while driving a commercial vehicle in the past 12 months.
\end{abstract}

Main outcome measure The likelihood of a crash associated with the use of substances containing caffeine after adjustment for factors including age, health disorders, sleep patterns, and symptoms of sleep disorders as well as exposures such as kilometres driven, hours slept, breaks taken, and night driving schedules.

Results Forty three percent of drivers reported consuming substances containing caffeine, such as tea, coffee, caffeine tablets, or energy drinks for the express purpose of staying awake. Only $3 \%$ reported using illegal stimulants such as amphetamine ("speed"); 3,4

methylenedioxymethamphetamine (ecstasy); and cocaine. After adjustment for potential confounders, drivers who consumed caffeinated substances for this purpose had a $63 \%$ reduced likelihood of crashing (odds ratio $0.37,95 \%$ confidence interval 0.27 to 0.50 ) compared with drivers who did not take caffeinated substances.

Conclusions Caffeinated substances are associated with a reduced risk of crashing for long distance commercial motor vehicle drivers. While comprehensive mandated strategies for fatigue management remain a priority, the use of caffeinated substances could be a useful adjunct strategy in the maintenance of alertness while driving.

\section{Introduction}

Long distance drivers of commercial motor vehicles routinely experience monotonous and extended driving periods in a sedentary position; this, combined with the disruption to circadian rhythms linked to the common requirement of night driving, has been associated with wake time drowsiness. ${ }^{12}$ Alertness is critical to safety for the driver and other road users as lapses of vigilance in relation to the task of driving can increase the likelihood of crashing. ${ }^{34}$ Many strategies are available to long distance drivers to maintain their alertness, and studies have identified positive benefits from the use of stimulants such as caffeine. ${ }^{56} \mathrm{We}$ investigated the effects of use of caffeinated substances on the likelihood of a crash among long distance drivers of commercial motor vehicles.

Caffeine is a psychostimulant that works by suppressing the innate adenosine mediated drive to sleep while activating arousal $^{78}$ and is one of the most commonly consumed stimulants worldwide. ${ }^{9}$ It has been shown to increase alertness in shift workers ${ }^{10}$ and improve the capacity for task performance. ${ }^{5}$ When used in excess, however, caffeine can also affect the quantity and quality of sleep, as sleep latency is prolonged and slow wave sleep is reduced. ${ }^{11}$ The effectiveness of caffeine for enhancing alertness in the performance of monotonous tasks has previously been shown in laboratory and driving simulator settings. ${ }^{5}$ Some authors have raised concerns about the methods used in these studies-namely, that the effects seen in comparisons of caffeine and placebo groups are simply an exaggerated response to caffeine withdrawal because of caffeine deprivation before the test and not caused by the substance alone. ${ }^{13}$ In contrast, a recent study used a field setting and found that a combination of caffeine and naps improved performance and alertness for night workers, ${ }^{14}$ and Mets and colleagues ${ }^{15} 16$ 
have described both driving improvement and increased alertness in a simulator setting while adjustment for average daily caffeine consumption to prevent withdrawal effects after administration of coffee.

A recent systematic review examined the influence of caffeine in the prevention of injuries caused by impaired alertness in shift workers. While the use of caffeine was recognised as an effective strategy for improving alertness, the findings were inconclusive in relation to any reduced likelihood of injury. ${ }^{10}$

Stimulant substances other than caffeine include nicotine and illegal substances such as amphetamine drugs in their various forms. Research regarding the use and influence of illegal stimulants in long distance drivers is limited but does suggest that their use is still occurring; and the self reported prevalence of illegal stimulant use among Australian long distance drivers has varied from $19 \%$ to $32 \% .^{17-19}$ Importantly, most drivers in these studies attributed their use to the need to maintain alertness while driving long distances. ${ }^{19}$ Mabbot and Harley ${ }^{18}$ suggested that efforts to reduce the use of illegal stimulant substances in these drivers would need to be introduced at the same time as equally effective countermeasures to maintain alertness on the road to prevent an increase in the incidence of crashes. None of these studies simultaneously explored the use of legal stimulant substances (such as caffeine) for the purpose of maintaining alertness.

We explored the use of caffeinated substances among a large population of long distance drivers of commercial vehicles and investigated the effect of use of legal stimulants in relation to the risk of crashing.

\section{Methods}

We conducted a case-control study between December 2008 and May 2011 across the Australian states of New South Wales (population 7.3 million) and Western Australia (population 2.3 million) ${ }^{20}$ Participants were long distance drivers whose vehicle mass was at least 12 tonnes tare weight (for example, B-doubles, semi-trailers, road trains). Case drivers had to have crashed their commercial vehicle while on a long distance trip (defined as at least $200 \mathrm{~km}$ from the driver's operating base). Potential case participants were excluded if there was a fatality associated with the crash, the driver of the commercial vehicle was seriously injured (defined as being admitted to hospital for two weeks or more or losing consciousness as a consequence of the crash), or if they were driving a passenger coach or bus. Control drivers also had to be on a long distance trip (at least $200 \mathrm{~km}$ from base) at the time they were approached at the truck stop for interview. Control drivers were deemed ineligible if they had been involved in a crash attended by police in the previous 12 months or if they were driving a passenger coach or bus. Sample size calculations for this study are described elsewhere. ${ }^{21}$

\section{Identification of case drivers}

Reports of crashes involving commercial vehicles that were attended by police were sent weekly to the study team from the police crash database in each state. Drivers of the commercial vehicle were then sent a letter of invitation to participate in the study in which the driver was assured confidentiality should they choose to take part. They were informed that their data would be de-identified once they had completed both the interview and the diagnostic sleep apnoea test overnight and no participants would be identifiable in any reports or papers arising from the study. A trained interviewer conducted the telephone interview. Over the study period, 1751 case drivers were identified and invited to participate in the study, of whom 895 were eligible. Of these, we recruited into the study 530 drivers who had been involved in a crash attended by the police (59\%).

\section{Identification of control drivers}

Control drivers were approached at one of 25 different truck stops across NSW and WA. Interview locations were chosen based on information from industry and road authorities regarding major trucking routes for long distance drivers in each state. Drivers of heavy vehicles were randomly approached while they stopped for a meal break; 891 were deemed eligible for inclusion in the study, of whom 517 consented and participated in a face to face interview (58\%). Interviews were conducted across all days of the week, times of day, and months of the year, and on 66 different interviewing occasions over the study period. As for cases, all controls were assured confidentiality (with anonymity after completion of the second part of the study as described above) to minimise selection bias.

\section{Measurement of risk factors}

State based teams of research interviewers, all trained by the study's project manager, conducted a 40 minute interview with all study participants. The interview included questions on drivers' characteristics such as demographics, anthropometry, medical conditions, and drugs. We also obtained information regarding health behaviours such as patterns of alcohol consumption ${ }^{22}$ and use of legal and illegal substances to maintain alertness; these questions related to consumption over the previous month. Health related behaviours such as levels of physical activity and sleep quantity, quality, and sufficiency were self reported, and participants were asked the standardised validated questions in the Epworth sleepiness scale ${ }^{23}$ to measure wake time sleepiness and the multivariable apnoea prediction inde ${ }^{24}$ to ascertain symptoms of the sleep disorder, obstructive sleep apnoea. All participants reported data related to employment and vehicle as well as a detailed summary of scheduling information outlining work hours, breaks, and rests over the three days before the crash (for cases) and the interview (for controls). We collected specific detail about the "index trip"- that is, the trip in which the driver crashed for cases and the last driving day before the interview for controls. Drivers who crashed were generally interviewed within four weeks after the crash, according to ethically approved notification timelines; attention was paid to ensuring interviews were conducted as soon as possible within this timeline to minimise recall bias.

To determine the consumption of caffeinated stimulant substances such as tea, coffee, energy drinks, or caffeine tablets, we asked drivers to specify what (if any) substances they consumed for the purpose of staying awake while they were driving during the previous month. Drivers were asked to describe consumption patterns over that month, in quantity and frequency. Amounts consumed were described in an open ended format and then coded based on guidelines developed by Addicott and colleagues, ${ }^{25}$ such that a "cup of coffee" or a "can of energy drink" contained $100 \mathrm{mg}$ of caffeine and a cup of tea or can of caffeinated soft drink contained $50 \mathrm{mg}$ of caffeine. Caffeine tablets purchased over the counter contain $100 \mathrm{mg}$ per tablet and were therefore coded as such. We combined the amounts with the drivers reported frequencies and then converted individual responses into three levels of consumption: "low," "moderate," and "high." Low caffeine users consumed less than $200 \mathrm{mg}$ a day, moderate caffeine users $200-400 \mathrm{mg}$ a day, and high caffeine users more than $400 \mathrm{mg}$ a day. ${ }^{25}$ To ascertain the use of illegal stimulants, we asked drivers to indicate what (if any) illegal drugs they had used over the previous month. The list contained commonly known stimulant 
substances such as "speed" (amphetamine), cocaine, and ecstasy (3,4 methylenedioxymethamphetamine). If drivers said they had used any of these substances they were then asked further detail about the frequency and amount over the same period.

\section{Statistical analyses}

We used unconditional logistic regression to calculate the likelihood of involvement in a crash in long distance drivers who consumed stimulant substances containing caffeine (coffee, tea, caffeinated soft drinks, caffeine tablets, energy drinks) as well as other substances such as illegal stimulant drugs for the purpose of staying awake while driving. We randomised a point in time (for instance, hour of the day) of the detailed "index trip" for controls to similarly represent the distribution of crashes in case drivers and to allow statistical comparisons across the index trip. We then used driving time and breaks taken on that trip until the time of the crash or the randomised point in time for controls to adjust risk estimates. We used logistic regression to estimate the effects of stimulant use on the risk of crashing, with adjustment for potential confounding factors. Independent variables were selected if they were shown to have a relation with the outcome variable in univariate analyses $(\mathrm{P}<0.2)$. We also examined the effect modification by distance travelled for the main exposure, as well as interactions between age and various independent variables in the model. The final multivariate unconditional logistic regression model was adjusted for driving exposure ( $\mathrm{km}$ travelled in the week before the crash and the week before the interview for controls), night driving (defined as the driver travelling more than half of the trip between $11 \mathrm{pm}$ and $5 \mathrm{am}$ ), and number of breaks taken during the index trip. Adjustment was also made for the average number of hours slept a night during the working week and over the month before the crash/interview and a history of a previous crash when driving a heavy vehicle that was attended by the police in the previous five years, as well as the state in which recruitment occurred.

All statistical analyses were performed with Stata version 11.0 (version 11.0 StataCorp, College Station, TX).

\section{Results}

Of the 1047 drivers recruited, almost all were men (99\%, $\mathrm{n}=1039)$. Case drivers were slightly younger than control drivers (mean (SD) 44.2 years (10.8) $v 46.1$ years (9.9); $\mathrm{P}=0.004)$. Only $162(15 \%)$ of all participants had a body mass index (BMI) in the normal range; 391 (37\%) were overweight and 494 (47\%) were obese. Significantly more control drivers were obese $(\mathrm{P}=0.047)$.

Controls had significantly more driving experience than case drivers (mean (SD) 19.9 (11.8) v 15.1 (11.9); $\mathrm{P}<0.001)$. Case drivers were more likely to have had at least one crash in the previous five years (excluding the current crash) $(\mathrm{P}=0.002)$. Drivers in NSW were significantly more likely to stop for a break at large truck stops or service stations with facilities for truck drivers than WA drivers $(585$ (69\%) v $92(46 \%)$; $\mathrm{P}<0.001)$, and WA drivers more likely to stop at a roadside rest area for their breaks than NSW drivers $(56$ (28\%) v 109 (12.8\%), respectively; $\mathrm{P}<0.001)$.

Table $1 \Downarrow$ outlines the relevant characteristics of the drivers by case and control status, with the unadjusted comparison. Table 1 also describes the drivers' responses to questions regarding consumption of caffeinated beverages and energy drinks for the purpose of staying awake while driving over the past month and use of illegal stimulants over the past month.
Behaviours significantly associated with crash risk at the univariate level include the consumption of caffeinated substances for the purpose of staying awake (odd ratio 0.34 , $\mathrm{P}<0.001$ ), as well as hours of sleep (reported as "on average over the past month" during work days, $1.29, \mathrm{P}<0.001$ ) (table $1 \Downarrow)$. We also examined other health behaviours such as exercise frequency and alcohol consumption in univariate models. While all drivers reported low rates of regular exercise compared with national estimate ${ }^{26}$ there was no difference between cases and controls (221 (41.7\%) v 230 (44.5\%), $\mathrm{P}=0.36)$ for frequency of physical exercise. Heavy cigarette smoking was significantly associated with crash risk univariately in the category of more than one pack a day (odds ratio $0.49, \mathrm{P}<0.001$ ). According to the categorisation model used for self reported caffeine consumption (including tea, coffee, caffeinated soft drinks, and caffeine tablets), high levels were consumed by 192 (37\%) of controls compared with 70 (13\%) of case drivers. High consumption of energy drinks was also reported by more controls $(71,14 \%)$ than cases $(34,6 \%)$.

Drivers were also asked whether they had experienced any difficulty staying awake while driving over the previous month and then asked to quantify the frequency. There was a significant difference in responses for this, with fewer case drivers than controls reporting difficulty staying awake while driving (182 $(34.3 \%) v 209(40.5 \%), \mathrm{P}=0.04)$. Drivers answering positively to any degree for this question were then asked to indicate if they would stop and have a nap if tired. Similar proportions of case drivers $(128(70 \%)$ and controls $(150,72 \%)$ suggested that they would pull over and nap if tired while driving $(\mathrm{P}=0.7)$.

Table $2 \Downarrow$ presents the results of the multivariate regression analysis exploring the association between the use of caffeinated stimulant substances and the risk of crashing for long distance drivers. Admission of the use of these substances for the purpose of staying awake while driving was different between case drivers and controls, with a significantly reduced risk of a crash associated with consumption of caffeinated substances; this association remained after adjustment for multiple confounders. While consumption of illegal substances showed no relation with the risk of crashing, the prevalence of drivers using such stimulants was small. Heavy cigarette smoking was significant in the univariate analysis, though this effect did not remain in the multivariate model. A history of a previous crash was also significant in univariate analyses, and this remained significant after adjustment for multiple confounders, such that having had a crash in the previous five years increased the risk of a crash by $81 \%$.

\section{Discussion}

In a large population of long distance commercial vehicle drivers, the consumption of caffeinated substances for the purpose of staying awake while driving was found to significantly protect against crashing after adjustment for multiple confounders. There was variation in reported caffeine consumption between the two states examined-New South Wales and Western Australia-and adjustment for this did not significantly alter the estimates. Use of illegal stimulants was not significantly associated with the risk of crash, though this could be because of the low reported prevalence of such use. These findings suggest that the consumption of legal stimulants such as caffeine could be of value within the context of a multi-faceted strategy to manage fatigue to reduce the propensity for crashes among long distance commercial drivers. 


\section{Comparison with other studies}

Our findings confirm those from previous research in which consumption of caffeine and energy drinks was shown to help in the maintenance of alertness for driving. ${ }^{57}$ Earlier research used computerised laboratory tasks to measure of sustained attention with and without caffeine consumption. ${ }^{28}$ Later studies that measured levels of alertness with caffeine versus placebo during prolonged driving in simulators were more comparable with the monotonous task of long distance driving. ${ }^{29}$ While previous studies predominantly compared a single dose of the same amount of caffeine with a placebo, ${ }^{529}$ our study provides new evidence for the influence of caffeinated substances, reported as consumed "for the purpose of staying awake," on prospective outcomes of driving performance such as crashes. Importantly, and confirming studies conducted in a simulated environment, our study provides direct evidence in a real world setting in which crashes can have severe and real life consequences. Although we found a distinct difference in consumption of caffeinated substance between case drivers and controls, the validity of the levels of consumption (or dose-response quantification) was subject to reporting bias and therefore was not modelled other than as a binary variable for which it was deemed robust.

Previous studies of stimulant use by drivers have focused on illegal substances such as amphetamines, with higher self reported consumption compared with that reported by drivers in the present study. ${ }^{18}{ }^{19}$ This could be a result of the roadside drug testing and associated enforcement initiated in 2008 in NSW and in 2007 in WA. A reduction in illegal stimulant use among commercial vehicle drivers has also been observed in the United States since the introduction of random roadside drug testing. ${ }^{30}$ The lower reported use of illegal substances in our study, however, could merely reflect a reticence by drivers to admit their drug use because of the awareness of tighter enforcement, despite being assured confidentiality for interview responses.

\section{Limitations}

While commercial vehicle drivers quantified their "usual" daily caffeine consumption over the past month "for the purposes of staying awake," they were not specifically questioned as to the timing of consumption or whether they consumed caffeine for other reasons nor were they provided any guide as to reporting of quantities and frequencies. Future studies would benefit from eliciting more information about the timing of consumption and precise amounts consumed, as well as the alertness levels at the time of and subsequent to consumption.

The exposure measures of interest were self reported and, as such, have limitations. It is notable, however, that for the quantification of habitual caffeine consumption and its effects, almost all previous studies have used self report. Importantly, the drivers in this study reported an average of 17 years' experience in their profession; we therefore considered that many of their behaviours were likely to be habitual. Despite the considerable variability in the self reported quantities of caffeinated beverages consumed as well as in consumption patterns, previous research has reported a reasonable degree of accuracy between self report and objective testing, albeit limited at the higher consumption levels. ${ }^{25}$ Taking this into account, we minimised the potential for reporting bias associated with the quantification of caffeine by only descriptively presenting the dose measures and not including them in the parsimonious model.
Given the need to undertake the study across two Australian states, there is potential for state level factors to influence our findings. For example, there are different legislative requirements between the states regulating work practices of long distance drivers, such as the fatigue management guidelines in NSW, which require all drivers to keep a work diary. This record can be interrogated at mandated truck stops across the state; in WA this does not apply. Such state specific factors could have influenced driver behaviour as well as recall of their behaviour, such as the number of breaks taken, hours driven, and possibly other self reported behaviours. By adjusting for these factors in the final model we have attempted to minimise what effect these biases could have on the interpretation of the study's findings. We cannot, however, determine the precise degree to which these factors might explain the counterintuitive nature of some findings.

\section{Conclusions and policy implications}

The finding that the consumption of caffeinated substances can significantly protect against crash risk for the long distance commercial driver has important implications for the improvement of fatigue management strategies for this and similar populations - a strong focus both for government and industry in Australia, particularly over the past decade. Recently renewed fatigue management guidelines for all commercial drivers ${ }^{25}$ imposed limits on the length of continuous driving periods, the objective being to "provide sufficient rest breaks" in a full day of driving. These guidelines also caution against the use of illegal stimulants to manage fatigue, though the consumption of caffeinated beverages is suggested as useful for improving alertness for a short time. Napping during breaks when tired is also strongly recommended; yet this is obviously not enforceable. In our study only $70 \%$ of drivers reported having stopped for a nap when tired, and, although we did not probe in detail regarding impediments to nap taking, the numbers suggest that it is not a strategy considered as favourable as others for the management of fatigue. While it is clear that taking breaks is a vital fatigue management strategy for long distance drivers, it is possible that the different activities undertaken during a break would contribute differently to a driver's fatigue or alertness level. The varying extent to which activities such as taking a nap, drinking a cup of coffee, or going for a short walk contribute to subsequent vigilance behind the wheel are not well understood and are therefore recommended for further study. In their recent systematic review of the role of caffeine in injury prevention, Ker and colleagues similarly concluded there is a need for a better understanding of the effects of caffeine relative to other countermeasures. ${ }^{10}$

Accountability for the occupational health and safety of the long distance driver is described in Australia as a "chain of responsibility" 25 that is shared by the regulator, employers and operators, loading managers, suppliers, and the drivers themselves. There are enforceable regulations that include driving and rest periods; there are also various non-enforceable but evidence based strategies that will help the driver to maintain the alertness required to drive safely. Our findings suggest that the consumption of caffeinated stimulant substances is associated with a significantly reduced risk of involvement in a crash for long distance drivers in Australia. While comprehensive fatigue management strategies for these drivers should consider the provision for adequate breaks and sufficient sleep and the promotion of regular exercise, the use and influence of caffeinated stimulants should be considered as an effective adjunct strategy to maintain alertness while driving. 


\section{What is already known on this topic}

Long distance commercial vehicle drivers routinely experience monotonous and extended driving periods in a sedentary position combined with shift scheduling they are at considerable risk of daytime fatigue

Long distance drivers have been known to use illegal stimulants to counter fatigue

Caffeine is a widely consumed legal stimulant, known to enhance alertness and task performance in simulated driving; real world studies with performance related outcomes such as crash are lacking in this population

\section{What this study adds}

Consumption of caffeinated drinks can reduce the risk of crashing among long distance commercial motor vehicle drivers by $63 \%$ compared with drivers who do not report taking caffeinated stimulants for the purpose of staying awake while driving

Contributions: LNS contributed to the design of data collection tools, collected data for the whole study, trained associate staff who collected data, wrote the statistical analysis plan, cleaned and analysed the data, and drafted and revised the paper. JE contributed to the design of data collection tools, trained associate staff, managed the project, and revised the paper. LM and RI contributed to protocol design, data collection tool design, analysis and interpretation of data, and revision of the paper. SB assisted in writing the statistical analysis plan and with the analysis and interpretation of data, and the revision of the paper. MS was principal investigator and conceived the protocol, guided data collection tool development, analysis and interpretation of data, and revision of the paper as doctoral supervisor of LNS. LNS is guarantor.

Funding: The study was funded by the Australian Research Council, the Australian Government Department of Infrastructure, Transport, Regional Development and Local Government, DiagnoselT, the National Transport Commission, Queensland Transport, the Roads and Traffic Authority of New South Wales and Main Roads in Western Australia. LNS is supported by Australian postgraduate awards industry grant (Australian Research Council). MS and RI are supported by NHMRC fellowships.

Competing interests: All authors have completed the ICMJE uniform disclosure form at www.icmje.org/coi_disclosure.pdf (available on request from the corresponding author) and declare: no support from any organisation for the submitted work; no financial relationships with any organisations that might have an interest in the submitted work in the previous three years; no other relationships or activities that could appear to have influenced the submitted work.

Ethical approval: This study was approved by the human research and ethics committees at the University of Sydney, NSW (No 10294) and Curtin University, WA, and informed consent was given by all participants.

Data sharing: No additional data available.

1 Jones I, Stein $\mathrm{H}$. Effect of driver hours of service on tractor-trailer crash involvement. Insurance Institute for Highway Safety, 1987.

2 McCartt A, Rohrbaugh J, Hammer M, Fuller S. Factors associated with falling asleep at the wheel among long-distance truck drivers. Accid Anal Prev 2000;32:493-504.

3 Connor J, Whitlock G, Norton R, Jackson R. The role of driver sleepiness in car crashes: a systematic review of epidemiological studies. Accid Anal Prev 2001;33:31-41.

4 Sharwood L, Elkington J, Stevenson M, Wong K. Investigating the role of fatigue, sleep and sleep disorders in commercial vehicle crashes: a systematic review. Australian College Road Safety $\mathrm{J}$ 2011;22:24-32.

5 Brice C, Smith A. The effects of caffeine on simulated driving, subjective alertness and sustained attention. Human Psychopharmacol 2001;16:523-31.

6 Philip P, Taillard J, Moore N, Delord S, Valtat C, Sagaspe P, et al. The Effects of Coffee and Napping on Nighttime Highway Driving. Ann Intern Med 2006;144:785-91.
7 Fredholm B, Battig K, Holmen J, Nehlig A, Zvartau E. Actions of caffeine in the brain with special reference to factors that contribute to its widespread use. Pharmacol Rev 1999;51:83-133.

8 Magkos F, Kavouras S. Caffeine use in sports, pharmacokinetics in man, and cellular mechanisms of action. Crit Rev Food Sci Nutr 2005;45:535-62.

9 Spiller G, ed. The methylxanthine beverages and foods: chemistry, consumption, and health effects. Alan R Liss, 1984

10 Ker K, Edwards P, Felix L, Blackhall K, Roberts I. Caffeine for the prevention of injuries and errors in shift workers. Cochrane Database Syst Rev 2010;5:CD008508.

11 Carrier J, Paquet J, Fernandez-Bolanos M, Girouard L, Roy J, Selmaoui B, et al. Effects of caffeine on daytime recovery sleep: a double challenge to the sleep-wake cycle in aging. Sleep Med 2009;10:1016-24

12 Horne J, Reyner L. Counteracting driver sleepiness: effects of napping, caffeine and placebo. Psychophysiology 1996;33:306-9.

13 Heatherley S. Caffeine withdrawal, sleepiness, and driving performance: what does the research really tell us? Nutr Neurosci 2011;14:89-95.

14 Schweitzer P, Randazzo A, Stone K, Erman M, Walsh J. Laboratory and field studies of naps and caffeine as practical countermeasures for sleep-wake problems associated with night work. Sleep 2006;29:39-50.

15 Mets M, Baas P, van Boven I, Olivier B, Verster J. Effects of coffee on driving performance during prolonged simulated highway driving. Psychopharmacol 2012;222:337-42.

16 Mets M, Ketzer S, Blom C, Van Gerven M, Van Willigenburg G, Olivier B, et al. Positive effects of Red Bull energy drink on driving performance during prolonged driving. Psychopharmacol 2011;214:737-45.

17 Howard M, Desai A, Grunstein R, Hukins C, Armstrong J, Joffe D, et al. Sleepiness, sleep-disordered breathing, and accident risk factors in commercial vehicle drivers. Am $J$ Resp Crit Care Med 2004;170:1014-21.

18 Mabbot N, Hartley L. Patterns of stimulant drug use on Western Australian heavy transport routes. Transportation Res 1999; $\mathrm{F}(2): 115-30$.

19 Williamson A. Predictors of psychostimulant use by long-distance truck drivers. Am J Epidemiol 2007;166:1320-6.

20 Australian demographic statistics. Canberra Australian Bureau of Statistics, 2011.

21 Stevenson M, Sharwood LN, Wong K, Elkington J, Meuleners L, Ivers RQ, et al. The heavy vehicle study: a case-control study investigating risk factors for crash in long distance heavy vehicle drivers in Australia. BMC Public Health 2010;10:162.

22 Department of Health and Ageing. Australian alcohol guidelines. Australian Government, 2011. www.health.gov.au/internet/alcohol/publishing.nsf/Content/guidelines.

23 Johns M. A new method for measuring daytime sleepiness: the Epworth sleepiness scale. Sleep 1991;14:540-5.

24 Maislin G, Pack Al, Kribbs N, Smith P, Schwartz A, Kline L, et al. A survey screen for prediction of apnea. Sleep 1995:18:158-66.

25 Addicott M, Yang L, Peiffer A, Laurienti P. Methodological considerations for the quantification of self-reported caffeine use. Psychopharmacol 2009;203:571-8.

26 Canberra National Information and Referral Service. National health survey: summary of results, 2007-2008. Australian Bureau of Statistics, 2009:1-64.

27 Horne J, Reyner L. Beneficial effects of an "energy drink" given to sleepy drivers. Amino Acids 2001;20:83-9.

28 Frewer $\mathrm{L}$, Lader $\mathrm{M}$. The effects of caffeine on two computerised tests of attention and vigilance. Human Psychopharmacol 1991;6:119-28.

29 De Valck E, Cluydts R. Slow-release caffeine as a countermeasure to driver sleepiness induced by partial sleep deprivation. J Sleep Res 2001;10:203-9.

30 Hall J, ed. Alcohol and other drug use in commercial transportation. International Council on Alcohol, Drugs and Traffic Safety Tri-ennial Conference. Adelaide, 1995

Accepted: 05 February 2013

\section{Cite this as: BMJ 2013;346:f1140}

This is an open-access article distributed under the terms of the Creative Commons Attribution Non-commercial License, which permits use, distribution, and reproduction in any medium, provided the original work is properly cited, the use is non commercial and is otherwise in compliance with the license. See: http://creativecommons.org/licenses/bync/2.0/ and http://creativecommons.org/licenses/by-nc/2.0/legalcode. 


\section{Tables}

Table 1/ Demographics, health characteristics, and consumption of stimulant substances in long distance commercial vehicle drivers who were recently involved in crash (cases) and control drivers who had not crashed in previous $\mathbf{1 2}$ months, with odds ratio for crash risk. Figures are numbers (percentage) of participants unless stated otherwise

\begin{tabular}{|c|c|c|c|c|}
\hline & Cases $(n=530)$ & Controls $(n=517)$ & Unadjusted odds ratio $(95 \% \mathrm{Cl})$ & $P$ value \\
\hline Mean (SD) BMI & $29.7(5.4)$ & $30.8(5)$ & $0.97(0.95$ to 0.99$)$ & 0.002 \\
\hline Overweight $^{\star}$ & $203(38.3)$ & $188(36.4)$ & $1.02(0.85$ to 1.39$)$ & 0.52 \\
\hline Obese† & $234(44.2)$ & $261(50.5)$ & $0.78(0.61$ to 0.99$)$ & 0.05 \\
\hline Mean (SD) hours of sleep & $7.22(1.3)$ & $6.76(1.4)$ & $1.29(1.17$ to 1.42$)$ & $<0.001$ \\
\hline Regular consumption of alcohol & $357(67.3)$ & $408(78.9)$ & $0.48(0.42$ to 0.73$)$ & $<0.001$ \\
\hline $\begin{array}{l}\text { Means (SD) No of standard drinks consumed per } \\
\text { occasion }\end{array}$ & $5.7(4.3)$ & $6.5(6.0)$ & $0.97(0.94$ to 0.99$)$ & 0.03 \\
\hline Regular exercise taken & $221(41.7)$ & $230(44.5)$ & $1.1(0.87$ to 1.43$)$ & 0.36 \\
\hline Mean (SD) days of exercise/week & $3.4(1.8)$ & $3.3(2.1)$ & $1.03(0.94$ to 1.13$)$ & 0.43 \\
\hline Previous crash $\ddagger$ & $119(22.5)$ & $75(14.7)$ & $1.67(1.21$ to 2.29$)$ & 0.002 \\
\hline Mean (SD) $\mathrm{km}$ driven in past week & 3178 (1585) & $4384(1701)$ & 0.99 (0.99 to 0.99$)$ & $<0.001$ \\
\hline \multicolumn{5}{|l|}{ Uses substance to stay awake: } \\
\hline No & $368(69.4)$ & $227(43.9)$ & 1 & \\
\hline Yes & $162(30.6)$ & $290(56.1)$ & 0.34 (0.27 to 0.44$)$ & $<0.001$ \\
\hline \multicolumn{5}{|l|}{ Caffeine consumption§: } \\
\hline None & $414(78.1)$ & $266(51.4)$ & - & - \\
\hline Low & $26(4.9)$ & $29(5.6)$ & - & - \\
\hline Moderate & $20(3.8)$ & $30(5.8)$ & - & - \\
\hline High & $70(13.2)$ & $192(37.1)$ & - & - \\
\hline \multicolumn{5}{|l|}{ Consumption of energy drinks: } \\
\hline None & $452(85.2)$ & $407(78.7)$ & - & - \\
\hline Low & $16(3)$ & $16(3.1)$ & - & - \\
\hline Moderate & $17(3.2)$ & $15(2.9)$ & - & - \\
\hline High & $34(6.4)$ & $71(13.7)$ & - & - \\
\hline Often/sometimes used illegal stimulant & $10(1.9)$ & $20(3.9)$ & $0.47(0.22$ to 1.03 & 0.06 \\
\hline
\end{tabular}

${ }^{*} \mathrm{BMI}>25-<30$.

$\dagger \mathrm{BMI}>30$.

$\ddagger$ One or more crashes when driving long distance in commercial vehicle that was attended by police in past five years.

$\S$ Caffeine in tea, coffee, caffeinated soft drinks, and caffeine tablets.

IIncluding speed, ecstasy, cocaine. 
Table 2| Associations between use of stimulant substances and crashes in long distance commercial vehicle drivers who were recently involved in crash (cases) and control drivers who had not had crashed in previous 12 months. Figures are numbers (percentage) of participants

\begin{tabular}{|c|c|c|c|}
\hline & Cases $(n=530)$ & Controls $(n=517)$ & Adjusted ${ }^{*}$ OR $(95 \% \mathrm{Cl})$ \\
\hline \multicolumn{4}{|c|}{ Uses caffeinated stimulant: } \\
\hline Not & $368(69.4)$ & $227(43.9)$ & 1.00 \\
\hline Yes & $162(30.6)$ & $290(56.1)$ & $0.37(0.27$ to 0.50$)$ \\
\hline \multicolumn{4}{|c|}{ Uses illegal stimulantsł: } \\
\hline Never† & $520(98.1)$ & $497(96.1)$ & 1.00 \\
\hline Often/sometimes & $10(1.89)$ & $20(3.87)$ & $0.68(0.27$ to 1.67$)$ \\
\hline Previous crash§ & $119(22.5)$ & $75(14.5)$ & 1.81 (1.26 to 2.62$)$ \\
\hline
\end{tabular}

*Adjusted for age, distance driven, hours of sleep, night driving, and breaks taken and state of crash/recruitment. †Reference category.

łlncluding speed, ecstasy, and cocaine.

§Police attended CMV driver crash, past five years, not including current crash for cases. 